\title{
THE INTRODUCTION OF AI IN THE CONSTRUCTION INDUSTRY AND ITS IMPACT ON HUMAN BEHAVIOR
}

\author{
Marte H. Schia ${ }^{1}$, Bo C. Trollsås ${ }^{2}$, Håkon Fyhn ${ }^{3}$ and Ola Lædre ${ }^{4}$
}

\begin{abstract}
The digital shift has arrived in the construction industry, with the aim of increasing the efficiency. However, how should the industry implement digital tools? And how should a human-technology relationship work? The purpose of this paper is to illuminate how the construction industry can close the gap between the potential benefits and the harvested benefits of implementation of AI. This paper presents research based on a comprehensive literature review, a case study of a construction project in Norway, and three external interviews. The case study consists of a document study and seventeen semi-structured interviews.

The experiences established through this research indicate that it is possible to gain experience from the implementation of basic digital tools when implementing advanced technology, such as artificial intelligence (AI). When come at AI, the human-AI trust will be the most decisive factor for a successful implementation. This paper constitutes a piece of pioneer work, as it investigates the implementation of AI, and how humans and technology should work together.

This research is limited down to one case study as well as three digital tools. To extend the research it is recommended to discuss the adaption of AI on premise of the users, collect more empirical data and look into experiences done by other industries.
\end{abstract}

\section{KEYWORDS}

Collaboration, Commitment, Trust, Digitization, Artificial Intelligence

\section{INTRODUCTION}

The construction industry is currently experiencing transformation from traditional, hierarchically organized construction sites to digital and more autonomous ones. A digital shift is taking place, and the development of the digital is advancing so fast that the industry struggles to keep up (Harty et al. 2015). This paper constitutes to a piece

1 M.Sc. student, Department of Civil and Environmental Engineering, Norwegian University of Science and Technology (NTNU), Trondheim, Norway, +47 95076 760, martehsc@stud.ntnu.no

2 Planner \& Business Development VDC, AF Gruppen, Oslo, Norway, +47922 64865 , bochristian.trollsas@afgruppen.no

3 Senior Researcher, Department of Social Research, Norwegian University of Science and Technology (NTNU), Trondheim Norway, +47 91743 561, hakon.fyhn@ntnu.no

4 Associate professor, Department of Civil and Transport Engineering, Norwegian University of Science and Technology (NTNU), Trondheim, Norway +47 91189 938, ola.ladre@ntnu.no 
of pioneer work, as it investigates the implementation of AI. Implementation of AI is a relatively unexplored topic, especially within the construction industry. AI can automate several operations and increase the efficiency of the building process (Salehi and Burgueño 2018). This is aligned with the lean-mindset, where minimizing waste and maximizing value is central (Ballard and Howell 2003).

Based on the above mentioned, the purpose of this paper is to illuminate how the construction industry can close the gap between the potential benefits and the harvested benefits of implementation of AI. In order to find a way of closing the gap, the following two research questions has been developed.

- What are the potential benefits of implementing AI in the construction industry?

- How does the construction industry harvest the benefits of AI implementation today?

This research is limited to a case study of a building project located in Oslo, Norway, in addition to three external interviews. The case study is restricted to look at three digital tools. Based on the lack of published research regarding the implementation of AI, a document study and interviews has been chosen as case specific methods. It has also been necessary for the authors to take advantage of the research regarding implementation of lean, last planner system (LPS), lower levels of digitization and other industries. In the following, the article consists of a method chapter, a theoretical framework, findings and discussion and at the end a conclusion and further research.

\section{METHOD}

This study is based on a qualitative research method with the use of triangulation. Triangulation increases the research's validity (Yin 2009). Initially, a literature study has been performed in order to map current research on the topic. The empirical data collection consists of a single case study and three external interviews. Case study was chosen to obtain data from an ongoing project. External interviews were chosen with the aim of taking advantage of the experiences done by other industries. The following section describes the line of action of the methods used.

\section{LITERATURE STUDY}

The literature study was carried out using several acknowledged databases, journals, conference articles, articles, books and snowballing, in addition to recommendations from supervisors. A systematic searching strategy was developed, with keywords such as "Lean", "Last Planner System", "Implementation", "Digitization", "Culture", "Change", "Human Behavior", "Artificial Intelligence" in combination with various search functions and limitations such as "construction industry". The reliabilityobjectivity-accuracy-aptitude principle was used to evaluate the sources (NTNU 2019). Findings from the literature study are presented in the theoretical framework as a current state of research related to the topic of the study.

\section{EMPIRICAL DATA}

\section{Case Study}

Due to the lack of published research regarding implementation of AI, was case study chosen as a suitable method. The chosen case is Bispevika, a building project in Oslo, Norway. The project consists of approximately 7 years of construction and NOK 4-5 
billion (AF Gruppen 2019). Bispevika was chosen based on its non-traditional way of working regarding innovative processes and tools, and the fact that a project of that scale seems a perfect testing ground for implementations due to the possibility of learning and the possibility of having the same people improving it along the way. The Bispevika-mindset is based on a lean approach. To get a grip on the implementation of AI, it is chosen to look closer into the implementation of the three digital tools described in Table 1.

Table 1: Explanation of the chosen digital tools at Bispevika

\begin{tabular}{|c|c|c|c|}
\hline Tool & Description & $\begin{array}{c}\text { Level of } \\
\text { digitization }\end{array}$ & $\begin{array}{l}\text { Implementation } \\
\text { phase }\end{array}$ \\
\hline Touchplan & $\begin{array}{l}\text { A web-based construction collaborative tool, which can be seen as } \\
\text { a digital version of the tools in the LPS (Sticky notes and physical boards) } \\
\text { (Touchplan 2018) }\end{array}$ & Digitization & Late implementation \\
\hline Synchro & $\begin{array}{l}\text { A 4D digital construction platform. Gives the workers the } \\
\text { opportunity to visualize, discuss and collaborate in order to find all } \\
\text { possible constraints before executing (Synchro 2019) }\end{array}$ & Digitalization & $\begin{array}{c}\text { Midway } \\
\text { implementation }\end{array}$ \\
\hline ALICE & $\begin{array}{l}\text { An Al planning Software which uses the input from the users to, } \\
\text { create and optimize schedules based on the recipes created (ALICE } \\
\text { 2017) }\end{array}$ & $\begin{array}{c}\text { Digital } \\
\text { Transformation }\end{array}$ & Early testing \\
\hline
\end{tabular}

Document study and interviews was chosen as suitable methods to collect data. Two of the authors were also participating on a workshop with, and about ALICE, with the intent of acquire more knowledge about the tool.

\section{Document Study}

Mainly, three different project documents were used to support the data from the interviews and increase the authors knowledge regarding the project and ALICE. The document "Lean in Team Bispevika" is considered a guideline of understanding the culture and the way of working at the project. The documents "ALICE overview" and "Construction Information Model" can be considered as necessarily to acquire information about the complexity of ALICE.

\section{Interviews}

Seventeen semi-structured in-depth interviews, in addition to one pilot interview was carried out with interviewees from the main contractor and subcontractors. More specifically, 1 interviewee represented the company management, 3 represented the project management, 10 represented the operating department and 3 represented different subcontractors. The interviewees were people with different experiences, knowledge and mindsets regarding digitization.

The intention of the interviews has been to unveil the interviewees' opinions regarding the digital shift and its impact on the human behavior. A semi-structured technique was chosen to allow the interviewer to angle the questions and topics toward the relevance based on answers given by the interviewee (Yin 2009). Interviews was carried out with a prepared interview guide. The guide was developed in collaboration with supervisors/co-writers through a brainstorming of questions related to the research questions. Furthermore, all questions were grouped into categories, and the categories was named: Technology, Process and Culture. All interviews were recorded and completely transcribed. The analysis was conducted with inspiration from a step-wisedeductive inductive method, with the use of coding (Tjora 2017).

\section{External interviews}

With the aim of unveiling useful experiences considering implementation of AI in other industries, three external interviews were conducted. The three interviewees 
represented three different companies, further described in Table 2. All three interviews were conducted and analysed in the same way as the case specific interviews.

Table 2: Description of external companies

\begin{tabular}{|c|c|c|}
\hline Company & Description company & Description interviewee \\
\hline $\begin{array}{l}\text { Norwegian } \\
\text { open Al lab }\end{array}$ & $\begin{array}{l}\text { Research center that brings together various research efforts within } \\
\text { Al. Current key areas for the research are health, energy, ocean space, } \\
\text { digital economy and smart environments (NTNU 2019) }\end{array}$ & $\begin{array}{l}\text { Professor in Computer Science and Al. } \\
\text { Long experience within the development and } \\
\text { implementation of Al systems within the oil and } \\
\text { gas industry and fishing industry }\end{array}$ \\
\hline Inmeta & $\begin{array}{l}\text { One of the leading consulting companies within machine learning in } \\
\text { Norway (Inmeta 2019) }\end{array}$ & $\begin{array}{l}\text { Data scientist with specialization within } \\
\text { machine learning. Experience with development } \\
\text { and implementation of Al systems in the } \\
\text { healthcare industry }\end{array}$ \\
\hline Spacemaker & $\begin{array}{l}\text { Norwegian start-up company that has developed an Al based } \\
\text { technology that calculate and optimize a construction site (Spacemaker } \\
\text { 2019) }\end{array}$ & $\begin{array}{l}\text { Chief Operating Officer with experience } \\
\text { within implementation of Al in the design phase of } \\
\text { construction projects }\end{array}$ \\
\hline
\end{tabular}

\section{THEORETICAL FRAMEWORK}

The construction industry is claimed to be undigitized and with a low productivity development compared to other industries (Barbosa et al. 2017). However, research indicates that the right implementation of digitization will increase efficiency of the building process (Barbosa et al. 2017). More advanced technology such as AI is now entering the industry. With more or less no research considering the implementation of AI, it will be necessary to take advantage of research regarding the implementation process itself and combine it with the technical knowledge of AI.

\section{IMPLEMENTATION AND THE IMPACT ON HUMAN BEHAVIOUR}

Implementation consists of the process of putting into practice an idea, program, or a set of activities and structures new to people attempting or expecting to change (Fullan 2007). Implementation can be considered as a change process, with inputs and a wish about a specific output. Research shows that incomplete implementations are often a result of incomplete preliminary work, where organizations are focusing on "what" instead of "how" (Noonan 2017; Senior and Swailes 2010).

Organizations are about people, and people forms cultures. Senior and Swailes (2010) defines culture as the following: "What is typical for the organization, the habits, the prevailing attitudes, the grown-up pattern of accepted and expected behavior". An implementation can also be considered as culture work. Culture is deep-seated, and is, therefore, likely to be resistant to change (Senior and Swailes 2010).

Motivation to change is decisive when implementing something new, but how do people get motivated? The goal-setting theory is strongly supported by research (Arnold et al. 2010). Setting performance goals that are specific and challenging (but not impossible), and to which the person feels committed, is likely to improve their work performance, their self-efficacy and their willingness to commit to new challenges (Arnold et al. 2010). However, recent research indicates that clear performance goals is not always beneficial. When working with simple processes, performance goals are considered beneficial, but when the processes are complex the same measures could actually inhibit productivity (Kahneman 2011).

Implementing change can be a long-term process, and commitment to the process can be weakened. The achievement of short-term wins is therefore essential, both as a motivating factor and as a mechanism for tracking the process towards the longer-term goals (Senior and Swailes 2010; Arnold et al. 2010). Another central factor considering motivation is the "implementation drivers". The people which drives the development 
further. The implementation is likely to fail if only the implementation drivers are motivated, as collaboration is key to innovation. Collaborative attitude, relationships based on trust and responsibility towards each other will facilitate motivation (Senior and Swailes 2010).

Every change will require training (Roland and Westergård 2015; Arnold et al. 2010). A training strategy should be developed and consist of: Training needs analysis, training design and training evaluation. All three are equally vital (Arnold et al. 2010).

As above mentioned, an organization is about people and people that forms cultures. So, what will happen when an organization is formed of people and AI? Will the factors motivation, commitment, collaboration, and trust as the literature are mentioning as decisive for a successful implementation be affected? In order to answer this, it is necessary to look deeper into the possibilities and limitations of the AI.

\section{AI IN CONSTRUCTION}

Digitization, digitalization, and digital transformation are often referred to as the same. However, the words represent different levels of the digital. Digitization involves creating a digital version of analog information, such as checklists on your mobile device instead of paper. Digitalization refers to the second level, where machines can perform human-controlled processes, such as continuously updating your Building Information Model (BIM). Digital Transformation is the integration of digital technologies in a way that the organization is fundamentally changing how they operate (Clerck 2016).

$\mathrm{AI}$ is defined as the ability of a machine to mimic intelligent human behavior, thus seeking to use human-inspired algorithms for approximating conventionally challenging problems (Salehi and Burgueño 2018). The implementation of AI can be seen as a digital transformation. Bolton (2018) exhibit the leading advantages of AI for organizations to be the following: Improve end-users experience using the data it provides, automate tasks to allow humans to focus on work that will add value and reduce human errors and deliver services more quickly. This can be seen in relation with the lean principles where maximizing value, minimizing waste and increase the efficiency of the working process is central (Ballard and Howell 1998).

As well-known is the adaption of AI relatively low in the construction industry (Blanco et al. 2018). However, today there are some start-ups that offers applications relevant to scheduling and image recognition. Using historical figures in addition to human-inputs, algorithms can consider millions of alternatives for project delivery and continually enhance the schedules. Image recognition can identify unsafe workers and aggregate this data to inform future training and education priorities. However, any AIalgorithm is based on training rather than programmed, which means that algorithms needs a certain amount of data to perform at the level of humans. Obtaining large data sets is today considered as a limitation for many building companies (Chui et al. 2018).

AI may help the construction industry to overcome the industry's greatest challenges, including costs, scheduling and safety (Blanco et al. 2018). However, is the construction industry in the starting phase of the digital transformation, and few projects have actually implemented AI. A central question considering the implementation will be how to get a successful human-AI collaboration? 


\section{HUMAN-AI COLLABORATION}

The digital shift introduces new ways of working, where humans and technology have to cooperate. Traditional culture classifies humans and technology as separate entities (Carpenter et al. 2018). However, the last years technological development have resulted in advanced automation that can respond better than a human in specific situations (Abbass 2019). Humans can't compete with AI regarding analysis of data, information and knowledge, likewise AI cannot compete with a human's ability of pedagogy, creativity, visions and ethics (Carpenter et al. 2018).

As a successful human-human cooperation requires defined tasks and responsibility, a human-AI cooperation will require the same. Although, the tasks and responsibility are distributed between the human and AI, it can be difficult for the human to trust the AI output. How AI arrives at a particular prediction and recommendation, and further discern whether it is a good solution or not? This is one reason that adoption of some AI tools remains low in application areas where explainability is useful or indeed required (Chui et al. 2018).

Trust blend a complex array of interactions factors including attitude, beliefs, control, emotion, risk and power (Abbass 2019). Stated that AI do not have attitude, beliefs or emotion in addition to the human lack of understanding, may cause it hard for a human to trust the output. The human is in a vulnerable situation. However, if vulnerability is defined as f (capability, opportunity, intent), and one assume that the AI's intent is aligned with the human's intent, humans can through training learn AI's capabilities and opportunities. In other words, it is possible to increase the transparency, and further the trust, by educating humans about what AI can and cannot do, and where disruption might occur (Abbass 2019).

However, what happens if the human and AI disagree? Fluid interaction which allow the human to edit AI, and the AI to guide the human will be necessarily. The aim is to enable a collaborative exploration of the data that leads to common ground where both the AI and the human beliefs have been updated (Chander et al. 2018). An interaction that makes it possible for the AI to answer questions such as "Why do Ineed to re-plan at this point" will increase the human's ability to find biased data, understand and trust the AI (Fox et al. 2017) .

Another important factor regarding trust is time. If the machine operate reliably and predictable over a long time, humans will start to trust AI to the same degree they trust other humans (International Business Machines 2015). However, while it is possible to trust AI in a calculative, and a knowledge based way, it is difficult to reach a higher level of trust by identification (Lewicki and Bunker 1996) which seems to be reserved for fellow humans.

\section{FINDINGS AND DISCUSSION}

This section presents findings and discussion from the collected empirical data. With the aim of mapping where the construction industry is likely to be in the future considering AI and where the industry currently is regarding AI.

\section{THE POTENTIAL BENEFITS OF ARTIFICIAL INTELLIGENCE}

The digital shift is taking place, but it can be difficult to predict how the digitization will impact the construction industry. The findings are based on three external interviews, with high AI knowledge, in addition to nine case specific interviewees, with 
relatively low AI knowledge. The remaining eight case specific interviewees are not included, due to their lack of knowledge considering AI. The interviewees were asked an open question considering AI's future in the construction industry to map people's thoughts about construction's digital future. The question resulted in both examples of applications and possible impacts. During the analysis of the collected data, the applications emerged into three AI categories inspired by Lee (2018) and Blanco (2018), namely: Machine learning, Pattern recognition and Automation.

Machine learning consists of algorithms that can analyse data and develop new solutions based on historical figures in a way a human never would be capable of. Pattern recognition is the process of recognizing patterns by using a machine learning algorithm. Automation is the process where AI will be able to carry out tasks done initially by humans, or tasks that go beyond human's ability. Table 3 is structured after these three categories, further is the potential benefits of taking them in use discussed.

Table 3: Future application of AI presented by Team Bispevika

\begin{tabular}{|c|c|c|}
\hline Categories of Al & Application & Impact \\
\hline Machine learning & Scheduling & $\begin{array}{l}\text { Analysis of a large amount of data based on historical figures and human input. Makes it } \\
\text { possible to evaluate millions of scheduling options that take humans exponentially longer to } \\
\text { accomplish }\end{array}$ \\
\hline $\begin{array}{l}\text { Pattern } \\
\text { recognition }\end{array}$ & $\begin{array}{l}\text { Health, Safety and } \\
\text { Environment }\end{array}$ & $\begin{array}{l}\text { Predict/early detection of dangerous situations by using machine learning algorithms in } \\
\text { combination with pattern recognition }\end{array}$ \\
\hline $\begin{array}{l}\text { Pattern } \\
\text { recognition }\end{array}$ & Storing space & $\begin{array}{l}\text { A digital map that continuously shows the site and where it is possible to store materials } \\
\text { or machines. Will increase the predictability and efficiency on site }\end{array}$ \\
\hline $\begin{array}{l}\text { Pattern } \\
\text { recognition }\end{array}$ & $\begin{array}{c}\text { Detection of } \\
\text { unregistered people }\end{array}$ & $\begin{array}{l}\text { Using pattern recognition to detect people and find those who are not registered at the } \\
\text { construction site. Will increase the safety and possibility of larceny and criminal damage }\end{array}$ \\
\hline Automation & $\begin{array}{l}\text { Robots executing } \\
\text { dangerous work }\end{array}$ & $\begin{array}{c}\text { For example, work in the height, such as fire protection of steel beams. Will improve the } \\
\text { safety of humans }\end{array}$ \\
\hline Automation & $\begin{array}{l}\text { Self-driving construction } \\
\text { machinery }\end{array}$ & The use of robots and self-driving construction machinery will change work flow \\
\hline Automation & Quality assure work & $\begin{array}{l}\text { Robots that drives around the construction site scanning the site situation and compare it with } \\
\text { the BIM. This technology may save hundreds of hours spent of quality assuring work, both } \\
\text { considering the main- and subcontractors }\end{array}$ \\
\hline
\end{tabular}

All applications mentioned in Table 3 will impact the workflow and work tasks. In order to function optimally, the applications will require human-AI trust and humanAI collaboration. The industry is today putting trust in individual expertise over empirics. This is interesting as the human remember feelings more than the actual event as a whole (Kahneman 2011). AI will act on common sense with no underlying intentions. One interviewee says the following: "Some people have bad intentions, that an AI would never have. I would have been equally sceptical to a human being as an AI, owing to people's intentions". This makes it easier to trust AI. AI will also have the ability to explore several "What ifs" while humans cannot. This is positive considering the optimization of processes but on the other hand, the reason why there is a need for transparency, as described in the theoretical framework. AI will never have a bad day at work. A result will be increased predictability in workflow and quality.

However, good solutions will require the right amount of data, sufficient data, and the right variables. All nine case specific interviewees are mentioning that knowing that $\mathrm{AI}$ is based on the right data set or BIM will be a prerequisite for trusting the AI. Eleven out of twelve interviewees point out that visualization of why and how the output is made will increase their understanding of the causality and further increase the trust. One interviewee says the following: "I would have managed to rely on the AI if it visualized why and how the output was made. Exemplification makes people understand and learn." The need for explanation corresponds with the research described in the theoretical framework. As stated in the theoretical framework will interaction with AI be necessarily to get a functional collaboration. However, the 
majority of the interviewees sees it hard to imagine how communication with AI should work, especially if the AI and human disagree. Others can't imagine how they are supposed to disagree, if the input to the AI are based on human thoughts.

Considering the future, can it be beneficial to investigate the logistics and transportation industry and take advantage of their experiences considering machine learning and route optimization. The healthcare sector can contribute to experiences regarding pattern recognition. Experiences considering robotics can be taken from the manufacturing industry.

\section{HARVESTED BENEFITS OF ARTIFICIAL INTELLIGENCE}

This section is based on the seventeen case specific interviewees. To map the harvested benefits of artificial intelligence in Team Bispevika, it was chosen to investigate the implementation of three digital tools: Touchplan, Synchro and ALICE. The tools represent the three levels of digitization (digitization, digitalization and digital transformation), described in the theoretical framework. Table 4 and the following discussion will be structured after these three tools. The categories and sub-categories were developed in co-operation with supervisors as a foundation of the interview guide. A brainstorming and discussion of questions relevant for the research questions were conducted. Further, the different questions were emerged into Technology, Process and Culture and their following sub-categories as shown in Table 4. The three categories Technology, Process and Culture was inspired by Arnold (2010), and Roland and Westergård (2015) opinions regarding important implementation factors. An implementation can be seen as successful when all rows in Table 4 is marked.

Table 4: Today's situation regarding implementation of digital tools at Team

\begin{tabular}{|c|c|c|c|c|}
\hline Categories & Sub-categories & $\begin{array}{c}\text { Touchplan } \\
\text { (Digitization) }\end{array}$ & $\begin{array}{c}\text { Synchro } \\
\text { (Digitalization) }\end{array}$ & $\begin{array}{c}\text { ALICE } \\
\text { (Digital Transformation) }\end{array}$ \\
\hline Technology & $\begin{array}{c}\text { Knowledge } \\
\text { Training } \\
\text { Use } \\
\text { Person Independence }\end{array}$ & $\begin{array}{l}x \\
x \\
x \\
x\end{array}$ & $\mathrm{x}$ & \\
\hline Process & $\begin{array}{c}\text { Knowledge } \\
\text { Training } \\
\text { Routines } \\
\text { Person independence }\end{array}$ & $x$ & $x$ & \\
\hline Culture & $\begin{array}{l}\text { Visibility of the utility } \\
\text { Willingness to use } \\
\text { Sense of achievement } \\
\text { Ownership }\end{array}$ & $\begin{array}{l}X \\
X\end{array}$ & & \\
\hline
\end{tabular}

Touchplan is a web-based construction collaborative tool, which is a digital version of the tools in the LPS (Sticky notes and physical boards). There is currently no training strategy regarding LPS or the use of Touchplan. Most interviewees think that the technical training is enough, although there is no defined training strategy. The interviewees consider themselves as competent users that can operate independent of expert users. This can be seen in the context of Toucplan's easy user interface. It is important for a successful implementation to illuminate the benefits it has for each employee's daily work tasks. Team Bispevika manage this in a good way, which is reflected by the high willingness and motivation to use Touchplan. One interviewee tells the following: "Six month ago, the only thing I was able to do on a computer was opening an e-mail. Today I am using Touchplan to plan and coordinate my work tasks. It's fantastic."

However, it is notable, that the interviewees tell that they are feeling person independent, but at the same time feel no sense of achievement, ownership nor 
commitment. This underlines how dependent they are on implementation drivers, in combination with the lack of a well-defined strategy and concrete goals. It seems like the implementation drivers have clear thoughts about who needs to learn the different things (training analysis), but that there is a lack of getting it into a clear and tangible structure (training design) in addition to continuously evaluation with the workers involved (training evaluation). As stated in the theoretical framework will all three factors (analysis, design and evaluation) be decisive.

Synchro is a $4 \mathrm{D}$ digital construction platform, which gives the workers the opportunity to visualize, discuss and collaborate in order to find all possible constraints before executing. As with Touchplan, there is currently no defined training strategy regarding 4D planning nor training in Synchro. There are today few people that can operate the program, and the interviewees are mentioning lack of time and extra work as main reasons. The lack of time may be a result of prioritizing in connection with the fact that few interviewees see the upsides of learning Synchro. This seems to affect the willingness and motivation to learn, and further the sense of achievement, ownership and commitment. However, those who are using it are pointing out the benefits of using the $4 \mathrm{D}$ model to get a common understanding of work tasks. The model is especially helpful in terms of the diversity of languages on a construction site.

Touchplan and Synchro represent transparent technology. Transparent technology is technology where the human control how the input is transformed to an output. As a result of the human controlled process, which makes the process transparent, is the output reliable and easy to trust. However, there is no guarantee that the given output is the most optimized, for example in terms of progression, staffing and cost, as the human brain have limited capacity of seeing different possibilities.

ALICE is based on AI which analyze, optimize and provide an output, such as a schedule. The tool is currently in the testing phase, which is illuminated by the empty rows in Table 4. AI is today a "hot" topic and widely used term. As the understanding of AI is not general knowledge in the industry, it will be difficult for a worker to understand how ALICE arrives at the presented output, and further trust the output. Today's lack of historical figures makes ALICE dependent of human-input. Humans partially controls the output, which makes it easier to trust the output. However, with enough historical figures the human input will no longer be necessary. Trust between humans are a decisive factor for a good collaboration and will likewise be important for a good human-AI collaboration. All interviewees agree that the implementation of AI will require other knowledge and training than the lower degrees of digitization.

\section{CONCLUSION AND FURTHER RESEARCH}

This paper has the purpose of illuminating how the construction industry can close the gap between the potential benefits and the harvested benefits of the implementation of AI. The gap has been identified by looking into the construction's possible benefits of implementing AI and today's harvested benefits of AI.

The research has been conducted as a literature study, a case study of Team Bispevika and three external interviewees. It is possible to assume that the results can be generalized to other projects, as the chosen interviewees are persons with different positions, knowledge and attitudes considering the digital shift. Research done in this paper shows that technology, process, and culture are equally important to succeed with an implementation. Figure 3 and 4 illustrates the current implementation situation of 
Touchplan (digitization) and ALICE (digital transformation). Dark grey illustrates the required level for a successful implementation, and light grey illustrates that some factors are achieved but not everyone, while white illustrates that the required level for a successful implementation is far from reached.

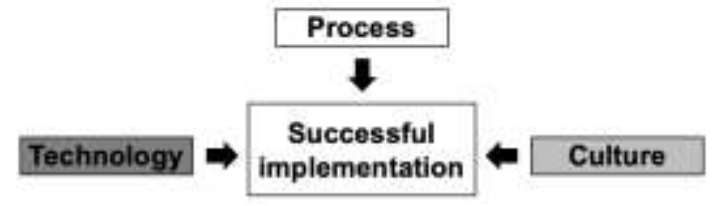

Figure 3: Implementation of Touchplan

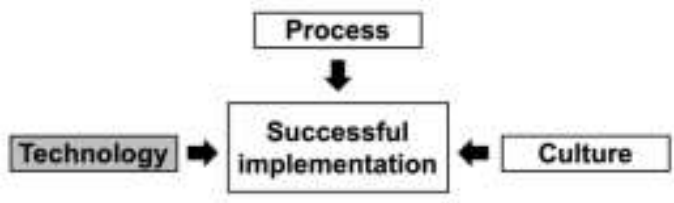

Figure 4: Implementation of ALICE

As shown in Figure 3, Touchplan and LPS are not considered implemented. However, Touchplan can be considered as a user-friendly tool, where Technology is sufficiently developed in order to succeed with the implementation. The expert users have managed to make engagement and positive attitude among the employees. This has further resulted in the willingness and motivation to learn and use the tool. The employees miss the sense of achievement and ownership, and as a result, is Culture marked light grey. The Process is marked white as there is no clear training strategy. As illustrated in Figure 4, ALICE is not fully implemented, and is currently in the testing phase. The AI-technology itself is present, but the absence of historical figures and transparency makes the Technology light grey. Process and Culture is marked white as there is no defined strategy of implementing ALICE nor a flexible, motivated and knowledgeable working culture. It can be concluded that AI has come to stay, and that there exist several benefits by implementing AI. However, several barriers must be broken in order to take advantage of the proposed benefits. Table 5 presents the factors concluded to be decisive for a successful implementation of AI.

Table 5: Factors considered essential for closing the gap between the current and future use of AI in the construction industry

\begin{tabular}{|c|c|}
\hline Experiences & Experiences and thoughts that can contribute to close the gap between the current and future use of $\mathrm{Al}$ \\
\hline Touchplan and Synchro & 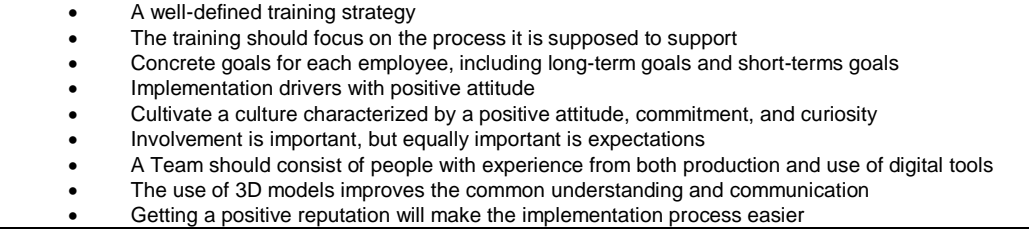 \\
\hline $\begin{array}{l}\text { Other factors considered } \\
\text { important. Based on } \\
\text { literature, empirical data } \\
\text { and the authors thoughts }\end{array}$ & 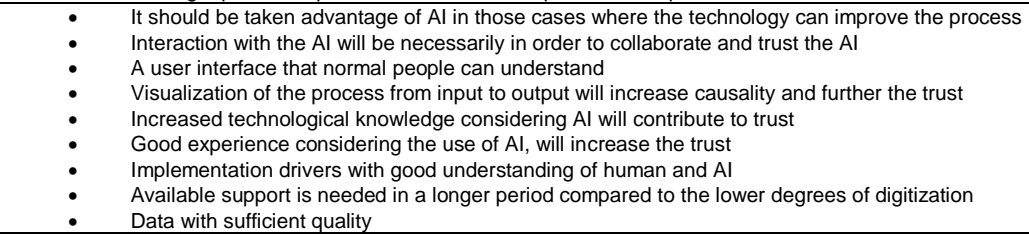 \\
\hline
\end{tabular}

The combination of human-AI trust and continuously optimizing a process makes it possible to increase the efficiency. Trust between humans and AI can more easily prevail when first established due to a lower possibility of conflict. AI can therefore be a supplement to improve a lean workflow.

AI technology differs from lower degrees of digitization as it has other prerequisites considering trust and collaboration with humans. It is recommended that further research discuss the adaption of AI on the premise of the users, collects more empirical data and investigates experiences done by other industries. 


\section{REFERENCES}

Abbass, H. A. (2019). "Social Integration of Artificial Intelligence: Functions, Automation Allocation Logic and Human-Autonomy Trust." Cognitive Computation, 10(48), 1-13.

AF Gruppen. (2019). "Boligutvikling Bjørvika." [Residential Development in Bjørvika] Boligutvikling $i \quad$ Bjørvika, <https://afgruppen.no/prosjekter/bygg/bispevika/> (Apr. 16, 2019).

ALICE. (2017). "ALICE - Construction planning, scheduling, and management powered by artifical intelligence." ALICE AI Construction, $</ / w w w . a l i c e t e c h n o l o g i e s . c o m>$ (Apr. 15, 2019).

Arnold, P. J., Randall, R., Patterson, P. F., Silvester, P. J., Robertson, P. I., Cooper, P. C., Burnes, P. B., Harris, D., Axtell, C., and Hartog, P. D. D. (2010). Work Psychology. Pearson Education Limited, Harlow, England.

Ballard, G., and Howell, G. (1998). "Implementing Lean Construction: Understanding and Action." Proc. of the 6th Ann.Conf. of the International Group for Lean Construction, Guaruja, Brazil.

Ballard, G., and Howell, G. (2003). "Lean Project Management." Building Research and Information, 31(2), 119-133.

Barbosa, F., Woetzel, J., Mischke, J., Riberirinho, M. J., Sridhar, M., Pearsons, M., Bertram, N., and Brown, S. (2017). "Reinventing construction: A route to higher productivity." Reinventing construction: A route to higher productivity, <https://www.mckinsey.com/ /media/mckinsey/industries/capital\%20projects $\% 20$ and $\% 20$ infrastructure/our\%20insights/reinventing\%20construction $\% 20$ through $\% 2$ 0a\%20productivity\%20revolution/mgi-reinventing-construction-executivesummary.ashx> (Feb. 8, 2019).

Blanco, J. L., Fuchs, S., Parsons, M., and Ribeirinho, M. J. (2018). "Artificial intelligence: Construction technology's next frontier." Artificial intelligence: Construction technology's next frontier, $<$ https://www.mckinsey.com/industries/capital-projects-and-infrastructure/ourinsights/artificial-intelligence-construction-technologys-next-frontier $>$ (Feb. 11, 2019).

Bolton, C., Machová, V., Kovacova, M., and Valaskova, K. (2018). "The power of human-machine collaboration: Artificial Intelligence, Business automation, and the smart economy." Economics, Management and Financial Markets, 13(4), 51-56.

Carpenter, S. A., Liu, C., Cao, W., and Yao, A. (2018). "Hierarchies of Understaning: Preparing for A.I." Learning and Collaboration Technologies. Design, Development and Technological Innovation, LCT, Las Vegas, USA, 20-39.

Chander, A., Wang, J., Srinivasan, R., Uchino, K., and Chelian, S. (2018). "Working with Beliefs: AI Transparency in the Enterprise." International Journal of Research in Engineering, Science and Management, 1(9), 4.

Chui, M., Manyika, J., and Miremadi, M. (2018). "What AI can and can't do (yet) for your business." What AI can and Can't do (yet) for your business, $<$ https://www.mckinsey.com/business-functions/mckinsey-analytics/ourinsights/what-ai-can-and-cant-do-yet-for-your-business> (Feb. 11, 2019). 
Clerck, J.-P. D. (2016). "Digitization, digitalization and digital transformation: the differences." $i$-SCOOP, <https://www.i-scoop.eu/digitization-digitalization-digitaltransformation-disruption/> (Feb. 22, 2019).

Fox, M., Long, D., and Magazzeni, D. (2017). "Explainable Planning."

Fullan, M. (2007). The New Meaning of Educational Change, Fourth Edition. Routledge, London, England.

Harty, J., Kouider, T., and Paterson, G. (2015). Getting to Grips with BIM. Routledge, London; New York.

Inmeta. (2019). “Inmeta." Inmeta, <http://inmeta.no/> (Apr. 15, 2019).

International Business Machines. (2015). "Building Trust in AI." IBM Cognitive What's next for $A I$, <http://www.ibm.com/watson/advantage-reports/future-ofartificial-intelligence/building-trust-in-ai.html> (Feb. 16, 2019).

Kahneman, D. (2011). Thinking, fast and slow. Farrar, Straus and Giroux, United States of America.

Lee, K.-F. (2018). AI Superpowers : China, Silicon Valley, and the New World Order. Houghton Mifflin Harcourt, Boston and New York, USA.

Lewicki, R. J., and Bunker, B. B. (1996). "Developing and Maintaining Trust in Work Relationships." Trust in Organizations: Frontiers of Theory and Research, SAGE, California, USA, 114-139.

Noonan, M. (2017). "Competency Models - What Are They Anyhow and What's the Big Deal?" Competency Models - What Are They Anyhow and What's the Big Deal?, <https://clevespace.com/stccg/wp-content/uploads/2018/04/CompetencyModels.pdf> (Feb. 11, 2019).

NTNU. (2019). "Finne kilder [Find sources]." NTNU, University page, <https://innsida.ntnu.no/wiki/-/wiki/Norsk/Finne+kilder> (Feb. 5, 2019).

NTNU. (2019). "Norwegian Open AI Lab." Norwegian Open AI Lab, $<$ https://www.ntnu.edu/ailab> (Apr. 15, 2019).

Roland, P., and Westergård, E. (2015). Implementering. Å omsette teorier, aktiviteter og strukturer i praksis [To put into practice theories, activities ans structures]. Universitetsforlaget, Oslo, Norway.

Salehi, H., and Burgueño, R. (2018). "Emerging artificial intelligence methods in structural engineering." Engineering Structures, 171, 170-189.

Senior, B., and Swailes, S. (2010). Organizational Change. Pearson, Edinburg, England.

Spacemaker. (2019). "Spacemaker.” Spacemaker, <https://spacemaker.ai/> (Apr. 15, 2019).

Synchro. (2019). "Synchro Software. The digital construction platform." Synchro Software. The digital construction platform. Clear, informed, reliable schedules start with 4D, <https://www.synchroltd.com/> (Apr. 15, 2019).

Tjora, A. (2017). Kvalitative forskningsmetoder $i$ praksis [Qualitative research methods in practice]. Gyldendal Norsk Forlag AS, Oslo, Norway.

Touchplan. (2018). "Construction Collaboration Software - Touchplan." Touchplan.io, <https://www.touchplan.io/> (Apr. 15, 2019).

Yin, R. K. (2009). Case Study Research: Design and Methods. SAGE, California, USA. 